\title{
LVI. On the ionization of various gases by the $\alpha$ particles of radium
}

\section{W.H. Bragg M.A.}

To cite this article: W.H. Bragg M.A. (1906) LVI. On the ionization of various gases

by the $\alpha$ particles of radium , Philosophical Magazine Series 6, 11:65, 617-632, DOI: 10.1080/14786440609463481

To link to this article: http://dx.doi.org/10.1080/14786440609463481

$$
\text { 曲 Published online: } 16 \text { Apr } 2009 .
$$

Submit your article to this journal $₫$

Џ Article views: 2

Q View related articles $\square$

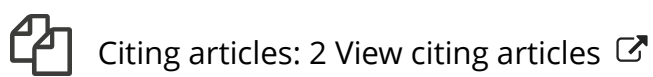




\title{
PHILOSOPHICAL MAGAZINE
}

\author{
AND \\ JOURNAL OF SCIENCE.
}

[SIXTH SERIES.]

MA Y 1906 .

LVI. On the Ionization of Various Gases by the a Particles of Radium. By W. H. BRaGG, M.A., Elder Professor of Mathematics and Physics in the University of Adelaide*.

TN a paper "On the Recombination of Ions in Air and other 1 Gases" (Phil. Mag. April 1906, p. 466), Mr. Kleeman and $I$ have described the preliminary steps of an enquiry into the total ionization produced in different gases by the $\alpha$ particle of radium, and the influence of physical conditions thereon. With the assistance of Mr. J. P. V. Madsen, B.Sc., I have made many experiments in continuation of this enquiry. It is necessarily a lengthy one, and in some respects difficult, so that on many of the points involved no definite conclusions are yet within reach. On others, results have been obtained which are, I think, of some interest and importance. In this paper I propose to describe the work which has been done; and, in addition, to make some reference to $(a)$ the magnetic deflexion of the $\alpha$ particle, $(b)$ its acquirement of a positive charge.

As described in the paper referred to, the total ionization of a gas can be measured in terms of the product of the coordinates of a certain point on the ionization curve. The true measure is, of course, the area between the curve and the axes of coordinates. But experiment shows that all ionization curves due to radium in radioactive equilibrium are of the same form, and differ from each other only in the

* Communicated by the Author.

Phil. Mag. S. 6. Vol. 11. No. 65. May 1906. 
application of some factor to all their ordinates or all their abscissæ. Thus the product of the coordinates of some standard point is proportional to the area of the whole curve, and may be taken as a relative measure of the total ionization.

In all the experiments to which $I$ am about to refer, the $\alpha$ particles cross at right angles a shallow ionization chamber whose upper electrode is a brass plate and the lower a brass gauze; the distance between the electrodes is about $3 \mathrm{~mm}$. An electromotive force of 300 volts is usually applied to the gauze, giving an electric force of 1000 volts per $\mathrm{cm}$. This is generally sufficient to ensure saturation; that is to say, to avoid all errors due to diffusion, general recombination, and initial recombination. When it is insufficient, the proper correction is found and applied. The ionization chamber is enclosed within a vessel which is satisfactorily air-tight except at higher temperatures, and this again within an electric oven. The gas under observation can thus be subjected to various pressures and temperatures. In the case of such substances as benzene and carbon tetrachloride, a temperature of from $60^{\circ}$ to $90^{\circ}$ is necessary to ensure a convenient gas-density. There is, however, this drawback to the use of high temperatures, that the insulators begin to lose their efficiency, and the joints cease to be air-tight. I find it necessary to use glass as an insulator instead of sulphur, for the latter cracks under the unequal expansions due to alteration of temperature. In the case of vapours, a certain quantity of air usually finds its way into the apparatus, for, as just mentioned, the joints leak somewhat at the higher temperatures. The amount so entering is sometimes determined by opening a communication between the vessel and an evacuated bulb, and weighing the quantity of mixture drawn off. The bulb and connexions are placed within the oven, and communication is made by opening a pinch-cock worked by a key projecting ouiside the oven. In this way, condensation in cold tubes is avoided. This method is not always employed, for as soon as the stopping-power of the gas is sufficiently well known, the proportion of the mixture is much more easily, and I think at least as accurately, determined by observation of the range of the a particles therein.

The insulation leak is determined by measuring the deflexion of the electrometer first for ten seconds, and then for twenty. With no leak the latter should be double the former; this is never quite the case, and the correction factor can be obtained from a comparison of the two values. 
The factor to be applied to a ten-second leak varies from about 1.03 at $40^{\circ} \mathrm{C}$. to about 1.10 at $70^{\circ} \mathrm{C}$.; at $90^{\circ} \mathrm{C}$. it is much higher.

The total ionization is measured in terms of the product $R I$. The ordinate $R$ is the range of the $\alpha$ particle, due to that product of radium whose speed is next to that of $\mathrm{Ra} C$. In air at $760 \mathrm{~mm}$. pressure and $20^{\circ} \mathrm{C} ., \mathrm{R}=4.83 \mathrm{~cm}$. very nearly. The abscissa $I$ is the ionization produced in the chamber described when the radium layer is at a distance of $4.83 \mathrm{~cm}$. from the middle of the chamber : or, more correctly, it is proportional to the ionization that would be produced in a very shallow chamber at that distance. The effect is wholly due to the particles from $\mathrm{RaC}$, the chamber being out of range of all the others.

These two quantities $\mathrm{R}$ and $\mathrm{I}$ differ materially from each other in two respects. To take the less important consideration first, the former quantity lends itself readily to exact measurement, the latter does not. The range of the a particle in a gas can be measured to an accuracy of one or two per cent. by a few minutes' observation, and to a much higher degree with greater care: it is perhaps the easiest of the measurements made in these experiments. By far the greatest difficulties which I find in the determination of the stopping-power of a gas lie in the purification and analysis of the gas.

On the other hand, the abscissa $I$ is much more difficult to measure. It is affected by variation in the sensitiveness of the electrometer, by leakage through the insulators, by variation of the dimensions of the apparatus, and its true value is not given unless enough electric force is applied. None of these things affects the range. But it is not merely in the details of measurement that these two quantities differ. They appear as physical constants to be in distinct categories: so far, that is to say, as can be observed at present. The stopping-power of an atom is a constant of the atom, unaffecter by its association with other atoms in molecular structure, independent of pressure and temperature. In a paper by Mr. Kleeman and myself (Phil. Mag. Sept. 1905), we gave a list of the stopping-powers of various substances, and since then we have made many other experiments in the same direction. In no case have we found a departure from the additive law which was not within the errors of experiment. That is to say, the range of the a particle in a given gas can always be predicted from the composition of the gas molecule. Not only so, but the stopping-powers of the various atums are very nearly proportional to the square roots of their 2 S 2 
weights, so that a simple, if approximate, law covers all the phenomena. It even seems justifiable now to go one step further. If the list in the paper quoted be examined, or the more comprehensive list in Table $A$, it will be found that the

TABLe A.

Table of Stopping-powers of various Gases.

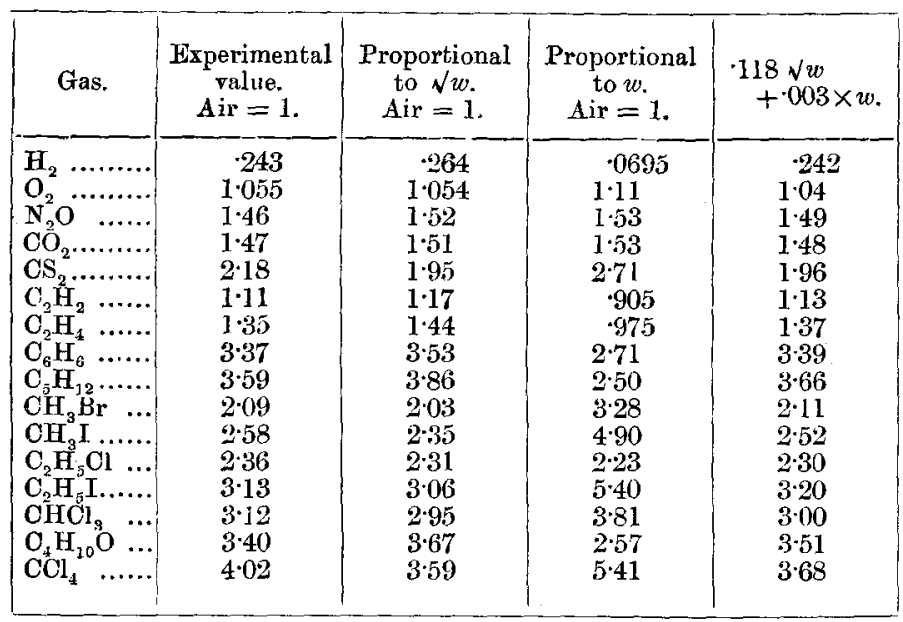

TABLE B.

Table of Stopping-powers of various Metals.

\begin{tabular}{|c|c|c|c|}
\hline Motal. & $\begin{array}{c}\text { Experimental } \\
\text { value. } \\
\text { Air }=1\end{array}$ & $\begin{array}{c}\text { Proportional } \\
\text { to } \sqrt{ } w \\
\text { Air }=1\end{array}$ & $\begin{array}{l}\text { Ratio of two } \\
\text { preceding } \\
\text { columns. }\end{array}$ \\
\hline Al & $1 \cdot 4 \overline{5}$ & $1 \cdot 37$ & $1 \cdot 06$ \\
\hline Fo .. & $2 \cdot 26$ & 1.97 & $1 \cdot 15$ \\
\hline $\mathrm{Ni} \ldots \ldots \ldots \ldots$ & $2 \cdot 46$ & $2 \cdot 20$ & $1 \cdot 12$ \\
\hline $\mathrm{Cu} \ldots \ldots \ldots \ldots$ & $2 \cdot 43$ & $2 \cdot 10$ & $1 \cdot 16$ \\
\hline $\mathrm{Ag} \ldots \ldots \ldots \ldots$ & $3 \cdot 17$ & $2 \cdot 74$ & $1 \cdot 16$ \\
\hline $\mathrm{Sn} \ldots . . . . . . .$. & $3 \cdot 37$ & 288 & $1 \cdot 17$ \\
\hline Pt $\ldots . . . \ldots \ldots . .$. & $4 \cdot 16$ & 368 & $1 \cdot 13$ \\
\hline An ............. & $4 \cdot 45$ & 370 & $1 \cdot 20$ \\
\hline $\mathrm{Pb} \ldots . . . \ldots \ldots . .$. & $4 \cdot 27$ & 378 & $1 \cdot 13$ \\
\hline
\end{tabular}

The last column of Table $A$ shows the application of the formula $a \sqrt{ } w+b w$ : the agreement with the second column is very close, considering that only two constants are used, and one of these is of little importance excepi in the case of the heavier atoms. But the formula does not appear to apply to the metals where the stopping-power varies very nearly as the square root of the atomio weight. This is certainly a difficulty. 
stopping-powers are systematic even in their slight departure from the square-root law. For, whilst dependent mainly on the square roots of the weights, they have a leaning towards the weights themselves. We did not call attention at the time to this fact, for we thonght it might be a spurious effect. But it has appeared so regularly in all further determinations that it seems right to note it, and to attempt an explanation of its physical meaning.

If we assume the correctness of the explanation already giren of the square-root law, viz., that the $\alpha$ particle spends energy for the most part on tearing away electrons from their attachment at the edges of the atom disks, then the natural complement to this is the further assumption that electrons in all parts of the atom disk may be disturbed to vibration by the passage of the particle, which latter therefore spends a small amount of energy in simple proportion to the weight of the atom. If $w$ be the atomic weight, the stopping-power of an atom should therefore be capable of expression by the formula

$$
a \sqrt{ } w+b w ;
$$

where the former term is usually by far the most important. The close agreement of the figures in the second and fifth columns of Table A shows that this is very nearly the case.

As regards pressure and temperature, I have not yet found any effect produced by variation of these conditions. The quantity $\mathrm{RP} / \mathrm{T}$ appears to be a constant, $\mathrm{P}$ being the pressure and $\mathrm{T}$ the absolute temperature. This implies that the stopping-power of an atom or molecule is independent of $P$ and T. Examples of the fact that RP is constant whilst $T$ is constant are given in the paper "On the Recombination of Ions in Air and other Gases." The following experimental result will serve as an illustration of the fact that $\mathrm{R}$ is proportional to $\mathrm{T}$ when $\mathrm{P}$ is constant. The ionization vessel filled with air was raised to a temperature of $90^{\circ} \mathrm{C}$., the pressure being $763 \mathrm{~mm}$. R was then found to be 5.98. Now when $p=760 \mathrm{~mm}$., and $\mathrm{T}=20^{\circ} \mathrm{C}$., $\mathrm{R}=4.83$. And

$$
\frac{4 \cdot 83 \times 363 \times 760}{5.98 \times 293 \times 763}=1.005 \text {. }
$$

It has of course been pointed out by several observers that the ionization effects of radium are largely independent of pressure and temperature and of physical and chemical conditions generally.

This, however, does not cover the present statement, which refers to the stopping-power of the atom, a quantity 
which has not previously been the subject of measurement. so far as I am aware.

To sum up, the range of the $\alpha$ particle in a given gas is, in the first place easily measured, and in the second place is simply related to the constitution of the gas and independent of its state. It is a delightful contrast to some other radioactive quantities, and often gives a welcome foothold in difficult places.

The quantity $I$ is in quite a different class. It is much more difficult to measure accurately, as I have already described. But there appears to be a more important difference in that the total ionization of a gas is not simply dependent on the weights of the atoms of which it is composed. Molecular structure counts for something. Perhaps, also, the various atoms do not yield ions in simple proportion to the energy spent on them, but this point is not yet sufficiently clear.

An example of this want of uniformity has already been given in the paper to which reference has been made. It was shown that RI in ethyl chloride is much greater than RI in air. The difference must be yet a little greater than that shown, as no allowance was made for the small quantity of air mixed with the heavy gas. Again, RI in standard pentane (mostly $\mathrm{C}_{5} \mathrm{H}_{12}$ ) is nearly half as much again as in air, and the same is almost certainly true of benzene $\left(\mathrm{C}_{6} \mathrm{H}_{6}\right)$, but this vapour is harder to treat than pentane, since a high temperature is necessary. Generally speaking, the more complex gases yield the greater number of ions. But the yield does not depend only on the number of atoms in the molecule. Acetylene $\left(\mathrm{C}_{2} \mathrm{H}_{2}\right)$ yields 25 per cent. more than air, yet $\mathrm{CO}_{2}$ with only one atom less yields but 5 per cent. more; and ethylene $\left(\mathrm{C}_{2} \mathrm{H}_{4}\right)$ yields the same as acetylene, though it has two atoms more. Of course in the last case the atoms added are very light; and $\mathrm{H}_{2}$ itself has, according to my measurements, a slightly lower value (for RI) than air. Rutherford also found this to be the case.

On the other hand, the influence of complexity can be illustrated by the cases of acetylene and ethylene as compared with benzene and pentane.

In order to bring out the significance of these comparisons, it should be pointed out that the a particle spends exactly the same amount of energy in every gas (Bragg, Phil. Mag. Nov. 1905).

Thus in different gases different numbers of ions are 
produced for the same expenditure of energy. It is quite clear, however, that this does not imply that the $\alpha$ particle finds it easier to produce ions in some gases than others. For, if so, there would be some influence on the stoppingpower of atoms dependent on the number of ions produced. But the stopping-power is connected with the atomic weigbt by a simple law: the number of ions produced is not. Plainly the energy spent by an $\alpha$ particle in an atom and the resulting ionization are not directly connected : there is an intervening link.

Either the ions made by the $\alpha$ particle produce others in some cases, or some of the ions made never emerge from the atoms. There is something which prevents the simplicity of the law governing the expenditure of energy by the $a$ particle from repeating itself in the amount of ionization produced. I think it is increasingly clear from our experiments that there is a secondary ionization within the molecule itself. The ions first marle, or possibly X-ray pulses accompanying ionization, have, in some cases, enough energy to make fresh ions before leaving the molecule. Thus, for example, one molecule of $\mathrm{C}_{6} \mathrm{H}_{6}$ is found to rob the a particle of just as much energy as three molecules of $\mathrm{C}_{2} \mathrm{H}_{2}$. But more ions are made out of the one $\mathrm{C}_{6} \mathrm{H}_{6}$ than out of the group of three acetylene molecules. This may be explained on the ground that the twelve atoms are crowded together, so that an ion projected under ionization from one of the atoms strikes one of the others with an energy undiminished by motion through the field of the positive from which it was originally separated, and so capable of detaching a second electron. In furtber consequence the ions emerging from a $\mathrm{C}_{6} \mathrm{H}_{6}$ molecule move more slowly than those from a $\mathrm{C}_{2} \mathrm{H}_{2}$, and are more liable to initial recombination. This is in agreement with experiment: it is far harder to saturate benzene than acetylene.

The secondary ionization would appear to take place within rather than without the molecule, because the amount of it does not depend upon the distance of the molecules from one another. The total ionization is independent of the pressure. It is certainly not due to the electric field, for if it were there would be no saturation value of the current.

I subjoin the details of two of the many experiments which Mr. Madsen and I have made. We hope to give a fuller description at some future time. 
Determination of Stopping-power, and of RI in Pentane.

Electrodes $3 \mathrm{~mm}$. apart (nearly). Volts applied $=300$. Temperature of apparatus $=35^{\circ} \mathrm{C}$.

Apparatus charged with vapour from standard pentane.

$\begin{array}{ccc}\begin{array}{c}\text { Distance from Ra } \\ \text { to middle of } \\ \text { ionization chamber. }\end{array} & \begin{array}{c}\text { Leak in } \\ \text { ten seconds. }\end{array} & \begin{array}{c}\text { Pressure inside } \\ \text { apparatus. }\end{array} \\ 2 \cdot 8 & 1982 & \\ 2 \cdot 9 & 1431 & 41 \cdot 15 \mathrm{~cm} . \\ 3 \cdot 0 & 1192 & \\ 3 \cdot 1 & 1171 & \\ 3 \cdot 2 & 1193 & 41 \cdot 15 \mathrm{~cm} . \\ 3 \cdot 3 & 1227 & \\ \text { Thin Cu foil over Ra } & 108 & \end{array}$

These being plotted, it is found that $R=2 \cdot 95, I=1044$, the copper leak having been deducted.

Thus $R=2.95$ in this mixture of pentane and air, at a pressure of $41.15 \mathrm{~cm}$., and a temperature (observed) of $308^{\circ}$ (absolute). But at a pressure $760 \mathrm{~cm}$., and $293^{\circ}$ absolute,

$\mathrm{R}$ in air is $4 \cdot 83$. much as air.

A special set of readings at $3.2 \mathrm{~cm}$. is now taken, three for 10 seconds and three for 20 seconds. The means are 1196 and 2325 respectively. Comparing these, it is found that the 10-second reading should be multiplied by 1.03 in order to allow for leakage by the insulators.

Again, a set is taken with 600 volts between the plates, and it is found that the mean reading when the copper leak is deducted is 1134. At the same time the reading for 300 volts, copper leak being deducted, is 1088 . Thus saturation is nearly complete.

A quantity of the gas is now drawn over into an exhausted bulb, whose temperature (that of the oven) is 311 (absolute): the pressure is observed to be $34 \cdot 5$. The weight of this gas is $\cdot 2536 \mathrm{gr}$. It is then calculated from a knowledge of the capacity of the bulb that the mixture weighs $2 \cdot 22$ times as much as air. From this it is found that to every molecule of pentane there are -23 molecules of air, assuming the pentane molecule to weigh 2.5 times as much as air. If $s=$ stopping-power of pentane, we have therefore

$$
\begin{aligned}
& & \frac{\cdot 23+s}{1 \cdot 23} & =3 \cdot 14 ; \\
\therefore & & s & =3.59 .
\end{aligned}
$$


Various Gases by the a Particles of Radium.

Again

$$
\begin{aligned}
\mathrm{RI} & =2 \cdot 95 \times 104 \cdot 4, \\
& =308, \text { uncorrected } .
\end{aligned}
$$

Correcting for want of saturation,

$$
\begin{aligned}
\mathrm{RI} & =308 \times \frac{1134}{1088}, \\
& =321 .
\end{aligned}
$$

On the same day and under the same conditions RI for air $=231$. The leakage correction is found to be the same for both, and need not be applied. Now, as far as consumption of energy is concerned, .23 molecules of air are equivalent to $\cdot 23 / 3 \cdot 59$ molecules of pentane, $=\cdot 065$. Hence if all the energy had been sperit as pentane molecules, the value for $\mathrm{RI}$ would have been

$$
\text { Finally } \quad \begin{aligned}
1 \cdot 065 & \times 321-\cdot 065 \times 231, \\
& =342-15, \\
& =327 .
\end{aligned}
$$

$$
\frac{\text { Total ionization in pentane }}{\text { Total ionization in air }}=\frac{327}{231}=1 \cdot 41 \text {. }
$$

It is quite possible that the low density of the mixture $(2.22$ instead of the 2.5 of pure pentane) is due to an admixture of lighter and more volatile hydrocarbons. If it were so, the result as to the value of RI would not be affected in any essential way.

\section{Determination of Stopping-power and RI of Acetylene $\left(\mathrm{C}_{2} \mathrm{H}_{2}\right)$.}

Same conditions as previous experiment. Apparatus charged to atmospheric pressure with gas; when tested, gas was found to contain less than 2 per cent. of impurities. Temperature of apparatus $=37^{\circ} .5 \mathrm{C}$. Barometer $=763 \mathrm{~mm}$.

$\begin{array}{cc}\begin{array}{c}\text { Distance from Ra to middle } \\ \text { of ionization chamber. }\end{array} & \begin{array}{c}\text { Leak in } \\ \text { ten seconds. }\end{array} \\ 4 \cdot 2 & 1430 \\ 4 \cdot 3 & 1276 \\ 4 \cdot 4 & 1024 \\ 4 \cdot 5 & 818 \\ 4 \cdot 6 & 698 \\ 4 \cdot 7 & 688 \\ 4 \cdot 8 & 701 \\ 4 \cdot 9 & 698 \\ \text { Cu over Ra. } & 46 \\ \text { At } 5 \cdot 2 \text { for } 300 \text { volts net leak } & =688 \\ \text { and "600 , , , , } & =706\end{array}$


Plotting these values it is found that $R=4.57$, I (less copper leak) $=635$. Hence $\mathrm{RI}$, corrected for want of saturation, $=298$.

Hence,

$$
\frac{\text { Total ionization for } \mathrm{C}_{2} \mathrm{H}_{2}}{\text { Total ionization for air }}=\frac{298}{231}=1.29 \text {. }
$$

Also stopping-power

$$
=\frac{483}{457} \cdot \frac{760}{763} \cdot \frac{3105}{2930}=1 \cdot 11 .
$$

In the paper by Mr. Kleeman and myself, to which I have already referred, it was pointed out that Rutherford had found it more easy to obtain the saturation current from a gas when it was removed from the influence of the ionizing agent. We observed that this could be easily explained by supposing initial recombination to be completed before the gas was subjected to the electric field. It is nevertheless no essential feature of the initial recombination hypothesis that the act of recombination should take place within any set time. The one important point is that the recombination takes place between two ions originally forming parts of one molecule. It is quite conceivable that for a certain time the positive and negative unay remain "semi-detached," their recombination in suspense until precipitated by some change of conditions. Curiously enough Mr. Madsen, working in this laboratory, has not yet been able to repeat Prof. Rutherford's experiment; and his results point to a prolonged existence of these pairs. $\mathrm{He}$ finds it hard to saturate a mixture of air and ether vapour which has been ionized by radium and then drawn away into a separate ionization chamber. It is not easy to reconcile this result with that of Prof. Rutherford: and it will be necessary to repeat the experiment under varying conditions. The point is one of considerable interest, for the existence of these pairs would help to explain much of the mechanism of phosphorescence. They would appear to be connected with the clusters of J. B. B. Burke, which were produced by ionization, gave rise to phosphorescent glow, contained energy, yet were not electrified. It is of interest in this connexion that the photograph which 
Sir William and Lady Huggins made of the phosphorescent glow of radium showed the bands of the gas in which the salt was imbedded. Rutherford also has shown that the $\alpha$ particle can no longer cause phosphorescence when it has lost its power of ionization.

\section{The Magnetic Deflexion of the a Rays.}

In the Physikalische Zeitschrift for Oct. 15th is a paper by M. Becquerel, " Ueber einige Eigenschaften der $\alpha$ Strahlen des Radiums." The author discusses the theory that the a rays gradually lose their velocity as they spend their energy on the ionization of the media through which they pass, a theory which I put forward about two years ago *, and which has the support of much experimental evidence accumulated by Prof. Rutherford + , and by Mr. Kleeman and myself $\neq$.

He maintains that the theory is unsuccessful in explaining the experiments which he has himself performed, and in particular he describes one experiment which he has devised as a crucial test and which he considers to show that the theory is incorrect.

It is as follows (loc. cit. p. 688) :-

The rays from a small quantity of radium salt are allowed to stream upwards through a narrow slit and fall upon a photographic plate. A powerful magnetic field deflects them slightly to one side. The field is reversed when the experiment is halfway through, and as a result two images of the slit appear, slightly separated, upon the plate. Now, M. Becquerel covers half the slit with a thin sheet of aluminium; and according to the theory which I have advanced, the $\alpha$ rays which pass through the sheet are thereby retarded. Consequently, M. Becquerel argues, these a rays should be more bent to one side than those which have not passed through the aluminium, and the images on the plate should show a break, the lines being more widely separated in one half of the picture than in the other.

But M. Becquerel is under a misapprehension on this point. Paradoxical as it may appear at first sight, no such

* Australasian Association for the Advancement of Science Report, Dunedin, January 1904.

+ Phil. Mag. July 1905.

$\ddagger$ Phil. Mag. Dec. 1904 and Sept. 1905. 
break ought to appear, and the photographic result is quite in accordance with the theory that the particles lose speed as they pass through matter.

In order that this may be clear, it is necessary first to consider the order of the deflexions of the $a$ rays in the magnetic field, on the various theories that have been proposed.

Suppose that an $\alpha$ particle is projected from $O$ in the direction $O N$ with velocity $v_{0}$, and that the action of a field $\mathrm{H}$ causes it to describe the curved path OA.

In the first place, let the velocity be constant throughout, and the path be therefore circular as $M$. Becquerel supposes. Then, since the curvature is small, $\mathrm{AN}=a^{2} / 2 \rho$, where $\mathrm{ON}=\alpha$, and $\rho$ is the radius of curvature.

Fig. 1.

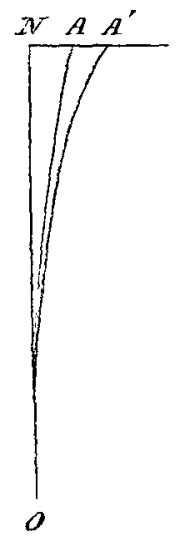

$$
\therefore \quad \mathrm{AN}=\frac{\mathrm{H} e}{m v_{0}} \cdot \frac{a^{2}}{2} .
$$

In the second place let the velocity diminish as the distance from $\mathrm{O}$ increases : and let us take the extreme case, where the velocity vanishes at a distance $a$ from $O$. Let the path in this case be $\mathrm{OA}^{\prime}$. It does not make very much difference what law of diminution of velocity we adopt: let us suppose, as my experimental results seem to indicate, that the particle spends its energy at a rate which is inversely proportional to the square of its speed. In this case,

$$
\frac{1}{2} m \frac{d v^{2}}{d s}=k v^{-2},
$$

$s$ being measured from $\mathrm{O}$, and therefore,

so that

$$
v^{4} \propto(a-s),
$$

$$
\frac{v^{4}}{v_{0}^{4}}=\frac{a-s}{a} \text {. }
$$

Thus

$$
\rho=\frac{m v_{0}}{\mathrm{H} e}\left(1-\frac{s}{a}\right)^{\frac{1}{4}},
$$

and we obtain easily that, if $\rho=d s / d \psi$,

$$
\psi=\frac{4 a}{3}\left\{1-\left(1-\frac{s}{a}\right)^{3 / 4}\right\} \frac{\mathrm{H} e}{m v_{0}} .
$$


Now, provided that $\int \psi d s$ is small, this quantity is very nearly equal to $\mathrm{A}^{\prime} \mathrm{N}$, the total deflexion of the ray.

But this integral

$$
\begin{aligned}
& =\int_{0}^{a} \frac{4 a}{3}\left\{1-\left(1-\frac{s}{a}\right)^{3 / 4}\right\} d s \cdot \frac{\mathrm{H} e}{m v_{0}} \\
& =\frac{\mathrm{H} e}{m v_{0}} \cdot \frac{4 a^{2}}{7} ;
\end{aligned}
$$

and this quantity is very small, since it is only slightly greater than $\mathrm{AN}$.

Finally then we have that

$$
\mathrm{A}^{\prime} \mathrm{N} / \mathrm{AN}=8 / 7 .
$$

It is easily found that if we had supposed the particle to spend its energy uniformly along its path, we should have obtained the result $\mathrm{A}^{\prime} \mathrm{N} / \mathrm{AN}=4 / 3$.

It will thus be clear that, on any reasonable hypothesis as to the particular law of diminution of velocity, the actual path of the particle differs very little from a circle. In the extreme case which I have considered, the small deviation therefrom at the end of the path is small compared with the widths of the images in M. Becquerel's photograph. If the particle ceases to ionize whilst its velocity is still great, as has been shown by Prof. Rutherford, the variation is still less.

Let us now consider the circumstances of M. Becquerel's experiment.

As a first approximation, suppose the widths of the groove containing the radium salt and of the slit to be negligible.

If no magnetic field is acting, all the $\alpha$ particles move in the vertical line $O N$. The range of the particles from $\mathrm{RaC}$ is very nearly $7.0 \mathrm{~cm}$.; from which it follows that the number which pass any given point $\mathrm{P}$ is proportional to the defect of OP from $7.0 \mathrm{~cm}$., or, in other words, that the number $n$ which end their flight on any unit of length of $O N$ is a constant. The other three groups of particles have, as their furthest distances of penetration, $4 \cdot 8,4 \cdot 2$, and $3 \cdot 5 \mathrm{~cm}$. respectively. Thus between $4 \cdot 8$ and $4 \cdot 2,2 n$ particles end their flight on each unit of length, between $4 \cdot 2$ and $3 \cdot 5$ the number is $3 n$, and from that point up to the radium $4 n$. The radium salt is supposed deep enongh to supply all these, i.e. its depth is taken to be at least $\cdot 001 \mathrm{~cm}$. Suppose now a powerful maynetic field to 
be brought into play, the direction of the lines of force being normal to the plane of the paper. The paths of the particles are curved to one side, and the curvature is greater the nearer the particle is to the end of its course. Let $O A$ and $O Q$ represent two such paths. Their separation from each other is considerably exaggerated in the figure. If all the paths were drawn the locus of $Q$ would be seen to be a curve, whose curvature in contrast to that of the path of any one particle would be greater the further the distance from $A$. This is in agreement with M. Becquerel's experiments, as I have previously pointed out*.

The width of the trace upon the paper of all the paths of the particles is very small, and is almost too fine to be shown on a diagram.

It is perhaps well to point out that there is no break in this trace at the critical points $4 \cdot 8,4 \cdot 2$, and $3 \cdot 5$. It is quite smooth from end to end. These points mark the extreme distance to which various bundles of rays penetrate; but the deflexion of an a ray

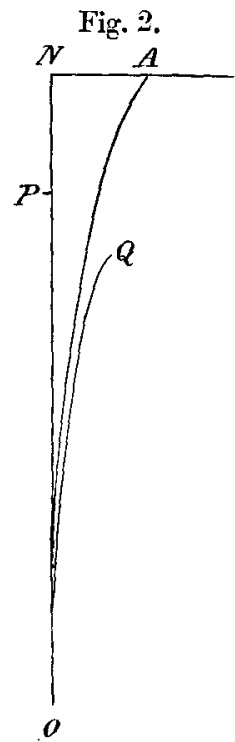
which ends its course at a given point is independent of the particular radioactive material from which it has come: the only varying characteristic of an $x$ particle is its velocity.

We must now take into account that the widths of the slit and the groove are not negligible, as is clearly to be seen from the photograph under consideration. There is consequently, so to speak, a large penumbra. Thus the trace upon the plane of the paper of all the $\alpha$ rays is such as is represented in fig. 3 , the deflexions being all exaggerated so as to be capable of depiction.

Now suppose an aluminium plate is placed, as in M. Becquerel's experiment, over the slit so that the $\alpha$ Fig. 3.

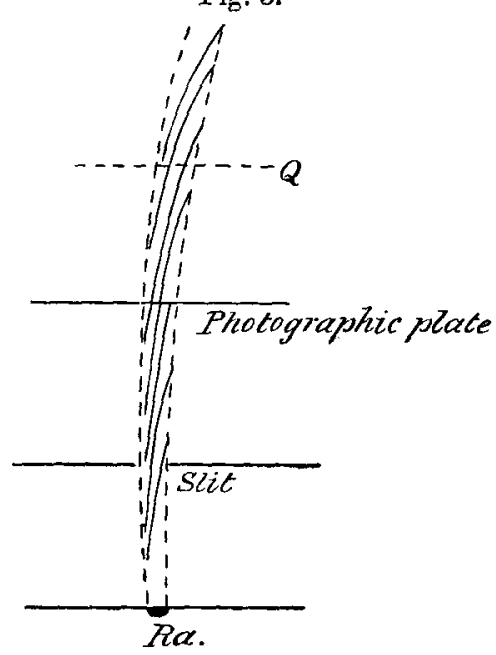

* Phil. Mag. Dec. 1904, p. 737 ; Jahrbuch d. Rad.u. Elektr. 1905, p. 14. 
particles have to pass through it on the way to the photographic plate. M. Becquerel supposes that there ought therefore to be an increased displacement of the photographic image. But this is not so. The path of any one $\alpha$ particle is slightly deflected, but the whole trace is not appreciably disturbed. The aluminium diminishes the range of every $a$ particle by the same amount, but the only result is to cut off all the rays which would have gone past a certain point, say $Q$, and to cause them to take the places of those rays which fell short of $\mathrm{Q}$; these latter being further shortened. This does not in the least affect the position of the outer edge of the trace upon the photographic plate; and though there must be a slight movement of the inner edge, so that the trace is somewhat narrower, the change is so small that it could not possibly be detected, as a glance at the photograph will show. Magnetic dispersion of the a rays does exist : it has been directly shown by Rutherford, and, as I think, indirectly by M. Becquerel's own experiments, in the peculiarities of the curvature of his photographic traces. But it could not be shown in the manner of the experiment which M. Becquerel now describes. That would be analogous to the search for evidence of the motion of the stars in the line of sight in the displacement of the visible spectrum as a whole; whereas the measurement to be made is of the displacement of the Fraunhofer lines in the spectrum, i.e. of one set of waves which can be isolated for consideration. It is here that Rutherford's experiment is differentiated from that of M. Becquerel. The former employed as a source of rays a wire coated with a thin layer of $\mathrm{RaC}$ emitting a particles of uniform velocity, which is analogous to confining one's attention in the star problem to waves of one length. Moreover, Rutherford passed his a particles for some considerable distance through a vacuum whilst yet under the influence of the magnetic field. Thus the evidence of the increase of curvature in their paths, originally caused by the loss of velocity in penetrating matter, was accumulated. But if, as in M. Becquerel's experiment, the path is in the air, then any appreciable increase of curvature closely precedes the cessation of all evidence of motion, and the result must be in any case almost beyond detection.

M. Becquerel remarks that there is no evidence in his photographs of the greater precision of the outer line of the trace, which I had anticipated. But the photograph which he now publishes show that there is too much penumbra for such an effect to be visible. 
632 Messrs. Hawthorne and Morton on Deflexions caused The Positive Charge of the a Particle.

Considerable discussion has recently taken place as to the mode in which the a particle acquires its positive charge. It has been pointed out more that once that it may be explained as the result of ionization by collision (Rutherford, Address to Congress at St. Luuis, 1904 ; Bragg, Phil. Mag. Dec. 1904), and that the same hypothesis will explain the deposit of the radium emanation on the negative electrode (Bragg and Kleeman, Phil. Mag. Dec. 1904). In the case of the emanation an explanation, which I understand to be similar, has been carefully worked out by Makower (Phil. Mag. Nov. 1905).

Rutherford has shown that the $\alpha$ particle is charged at the moment of leaving the radium salt. But I do not think that the resuit is in any way prejudicial to the collision theory. He evaporated a very weak solution of radium on a plate, and supposed that as a result he had an excessively thin layer, so that the particle made very few collisions before emergence. But when such deposits are examined under a microscope, it is seen that the salt is gathered in little heaps, and there is no true layer at all. The bulk of the $\alpha$ particles pass through hundreds of atoms before emergence, and there is ample opportunity for ionization by collision.

We find that the $\alpha$ particle spends energy in causing the expulsion of electrons from the atoms of any gas which it traverses. We find also (see Tables $A$ and $B$, above) that the expenditure of energy by the a particle follows the same law when the atoms are massed together in a solid. The solid must therefore be ionized, just as the gas is, and we should expect slow-moving electrons to be projected from $\mathrm{Ra}$. itself, and from both sides of any solid screen through which the particles pass. Surely this is the effect lately observed by J. J. Thomson, Rutherford, and others. This has been suggested by Soddy ('Nature,' March 1905).

LVII. Note on the Deflexions caused by a Break in an Overhead Wire carried on Poles. By W. HaWthonne, B.A., B.E., and W. B. Morton, M.A.*

T $N$ the modern conditions of electrical power-transmission 1 along overhead wires, it is a matter of practical importance to know what will happen in case a wire breaks. The relaxing of tension at one point will throw a one-sided pull on the range of poles on either side of the break; and it is essential for safety that the maximum deflexion thus produced should be below a definite limit.

* Communicated by the Authors. 